\title{
Intrinsic Anomalous Nernst Effect Amplified by Disorder in a Half-Metallic Semimetal
}

\author{
Linchao Ding $\odot,{ }^{1}$ Jahyun Koo, ${ }^{2}$ Liangcai Xu, ${ }^{1}$ Xiaokang Li $\odot,{ }^{1}$ Xiufang Lu, ${ }^{1}$ Lingxiao Zhao, ${ }^{1}$ Qi Wang, ${ }^{3}$ \\ Qiangwei Yin, ${ }^{3}$ Hechang Lei, ${ }^{3}$ Binghai Yan $\odot,{ }^{2, *}$ Zengwei Zhu $\odot,{ }^{1, \dagger}$ and Kamran Behnia $\odot^{4,5, \$}$ \\ ${ }^{1}$ Wuhan National High Magnetic Field Center and School of Physics, \\ Huazhong University of Science and Technology, Wuhan, 430074, China \\ ${ }^{2}$ Department of Condensed Matter Physics, Weizmann Institute of Science, 7610001 Rehovot, Israel \\ ${ }^{3}$ Department of Physics and Beijing Key Laboratory of Opto-electronic \\ Functional Materials \& Micro-nano Devices, Renmin University of China, Beijing, 100872, China \\ ${ }^{4}$ Laboratoire de Physique et Etude des Matériaux (CNRS/Sorbonne Université), \\ Ecole Supérieure de Physique et de Chimie Industrielles, 10 Rue Vauquelin, 75005 Paris, France \\ ${ }^{5}$ II. Physikalisches Institut, Universität zu Köln, 50937 Köln, Germany
}

(Received 11 July 2019; revised manuscript received 22 October 2019; published 24 December 2019)

\begin{abstract}
Intrinsic anomalous Nernst effect, like its Hall counterpart, is generated by Berry curvature of electrons in solids. Little is known about its response to disorder. In contrast, the link between the amplitude of the ordinary Nernst coefficient and the mean-free path is extensively documented. Here, by studying $\mathrm{Co}_{3} \mathrm{Sn}_{2} \mathrm{~S}_{2}$, a topological half-metallic semimetal hosting sizable and recognizable ordinary and anomalous Nernst responses, we demonstrate an anticorrelation between the amplitudes of carrier mobility and the anomalous $S_{x y}^{A}$ (the ratio of transverse electric field to the longitudinal temperature gradient in the absence of magnetic field). We argue that the observation, paradoxically, establishes the intrinsic origin of the anomalous Nernst effect in this system. We conclude that various intrinsic off-diagonal coefficients are set by the way the Berry curvature is averaged on a grid involving the mean-free path, the Fermi wavelength, and the de Broglie thermal length.
\end{abstract}

DOI: 10.1103/PhysRevX.9.041061

Subject Areas: Condensed Matter Physics, Magnetism, Topological Insulators

\section{INTRODUCTION}

Electrons in some solids do not flow along the applied electric field or thermal gradient, even in zero magnetic field. The phenomena, known as the anomalous Hall effect (AHE), the anomalous Nernst effect (ANE), and the anomalous thermal Hall effect, may be caused either by the geometric (Berry) curvature of Bloch functions or by the skew scattering of electrons off magnetic impurities. Distinguishing between these intrinsic and extrinsic origins (see Refs. [1,2] for reviews) has motivated numerous investigations during the past two decades.

Here we focus on the anomalous Nernst effect. The Nernst effect $S_{x y}$ is a transverse electric field generated by a longitudinal temperature gradient (in the absence of charge flow). It is directly accessible to the experimentalist and has

\footnotetext{
*binghai.yan@weizmann.ac.il

tzengwei.zhu@hust.edu.cn

*kamran.behnia@espci.fr
}

Published by the American Physical Society under the terms of the Creative Commons Attribution 4.0 International license. Further distribution of this work must maintain attribution to the author(s) and the published article's title, journal citation, and DOI. to be distinguished from the off-diagonal component of the thermoelectric tensor $\left(\alpha_{x y}\right)$. The latter is the transverse temperature gradient generated by a longitudinal charge flow (in the absence of electric field), which is often the immediate result of theoretical calculations. The two coefficients are intimately connected to each other [3].

A pioneer theoretical study of the intrinsic anomalous Nernst effect [4] argued that it depends on the magnitude of the magnetization in the host solid. The expected correlation between the two properties became the mantra of many of the numerous experimental studies of the ANE [5-16] in topological solids (for a review of such systems, see Refs. $[17,18]$ ). A prominent question, yet to be addressed, is the role of disorder in setting the amplitude of the ANE. Does this transport property depend on the mean-free path? If yes, since magnetization is a thermodynamic property, why? The ordinary Nernst effect (ONE) is expected to scale with mobility $[3,19]$. Experimental studies have not only confirmed this scaling across different systems, but also found that the magnitude of the ONE in a given solid increases as it becomes cleaner (bismuth and $\mathrm{URu}_{2} \mathrm{Si}_{2}$ are two prominent case studies) [3]. To the best of our knowledge, this has not been the case of the ANE.

In this paper, we show that this opportunity is offered for the first time by the newly discovered magnetic Weyl 
semimetal $\mathrm{Co}_{3} \mathrm{Sn}_{2} \mathrm{~S}_{2}$. This is a solid with a shandite structure, which becomes a ferromagnet below $180 \mathrm{~K}$ [20-22]. Theoretical calculations suggest that this semimetallic half-metal [23] with negative flatband magnetism [24] hosts Weyl nodes $60 \mathrm{meV}$ off the Fermi level [25]. Previous experiments have found a large AHE [25,26], a large ANE [12,14], and surface-termination-dependent Fermi arcs [27].

We report first on a significant improvement in the quality of $\mathrm{Co}_{3} \mathrm{Sn}_{2} \mathrm{~S}_{2}$ single crystals obtained by the chemical vapor transport (CVT) method. The improved quality shows itself in higher mobility compared to what was reported in previous studies $[12,14,22,25,26]$. This allowed us to perform a systematic study of five different crystals with different impurity concentrations. We find that, as expected $[3,19]$, the amplitude of the ordinary Nernst response $S_{x y}^{O}$ is proportional to mobility $\mu$; On the other hand, the amplitude of the anomalous Nernst effect $S_{x y}^{A}$ is proportional to the inverse of $\mu$. We argue then that the amplification of $S_{x y}^{A}$ by disorder reflects the fact that the anomalous transverse thermoelectricity $\alpha_{x y}^{A}$ depends on the Berry curvature (averaged over a reciprocal distance set by the thermal de Broglie wavelength), but not on the meanfree path. This is to be contrasted with the semiclassical $\alpha_{x y}$, which scales with the square of the mean-free path [3]. We show that according to both theory and experiment, the Fermi surface of $\mathrm{Co}_{3} \mathrm{Sn}_{2} \mathrm{~S}_{2}$ is complex. Finally, we determine the magnitude of the anomalous transverse thermoelectric conductivity $\alpha_{x y}^{A}$ in all five samples and find a qualitative agreement between theory and experiment with no need for invoking uncontrolled and unidentified extrinsic dopants invoked previously [14]. It is remarkable that the scaling between average mobility and the anomalous Nernst coefficient remains valid in spite of the multiplicity of Fermi surface pockets.

\section{SAMPLES WITH DIFFERENT MOBILITIES}

$\mathrm{Co}_{3} \mathrm{Sn}_{2} \mathrm{~S}_{2}$ crystallizes in the shandite structure, which consists of $A B C$ stacking of kagome sheets [see Fig. 1(a)]. A picture of a hexagonal sample with typical dimension of $1.3 \times 1 \times 0.02 \mathrm{~mm}^{3}$ is shown in the inset of Fig. 1(b). The in-plane resistivity $\rho_{x x}$ of the five samples (among a dozen measured [28]) is shown in Fig. 1(b). A kink at $177 \mathrm{~K}$ is visible in $\rho_{x x}(T)$. It indicates the Curie temperature and the opening of a gap in the spectrum of minority spins leading to the half-metallicity $[20,21]$. The residual resistivity ratio $[\mathrm{RRR}=\rho(300 \mathrm{~K}) / \rho(2 \mathrm{~K})]$ varies between 4 in the dirtiest sample (B5) and 21 in the cleanest (no. 31). Figure 1(c) compares the normalized resistivity (i.e., $\rho(T) / \rho(300 \mathrm{~K})$ of the samples with the data reported in four previous studies $[12,22,25,26]$. One can see that three of our chemical vapor transport samples display the highest RRR. In Fig. 1(d), the low-temperature resistivity is plotted versus $T^{2}$. One can see that disorder shifts the curves rigidly upwards and the
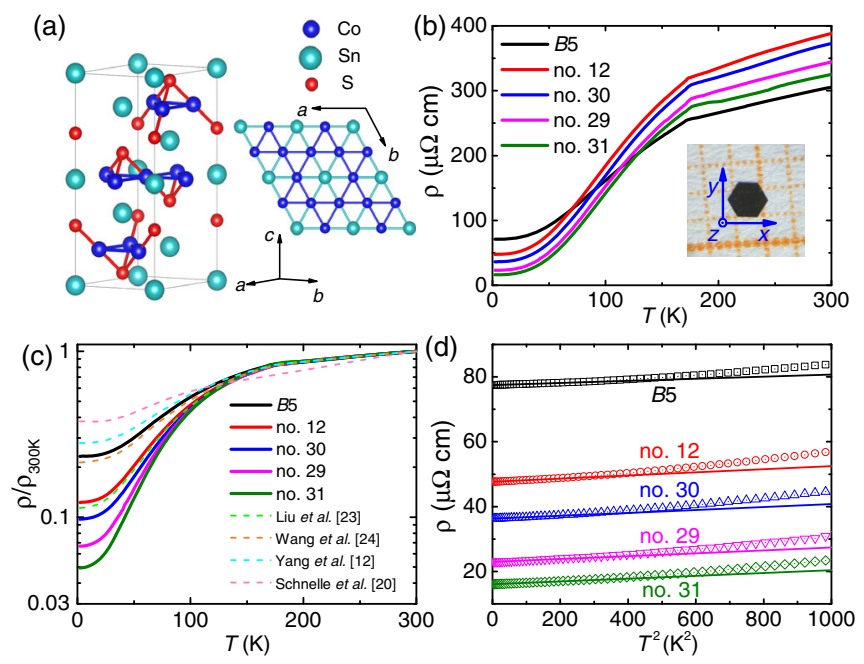

FIG. 1. Crystal structure, resistivity, and sample-dependent disorder. (a) Crystal structure of $\mathrm{Co}_{3} \mathrm{Sn}_{2} \mathrm{~S}_{2}$. (b) Temperature dependence of resistivity at zero field in five samples. The residual resistivity ratio (RRR) ranges from 4 to 21 . The inset shows an as-grown hexagonal-shape sample with a typical dimension of $1.3 \times 1 \times 0.02 \mathrm{~mm}^{3}$. The orientations of $x, y$, and $z$ are defined as [2 $\overline{1} \overline{1} 0],[01 \overline{1} 0]$, and [0001], respectively. (c) Comparison of resistivity, normalized to its room-temperature value between the samples used in this study and four previous reports. The previous RRR is comparable with the two dirtiest samples in this study. (d) $\rho$ as the function $T^{2}$ in our samples. The prefactor of $T$-square resistivity is similar in all four samples grown from chemical vapor transport method, but the difference is residual resistivity leads to an upward shift.

fivefold change in residual resistivity barely affects inelastic scattering. Indeed, the prefactor of the $T$-square resistivity [29] is the same (4.5 $\mathrm{n} \Omega \mathrm{cm} \mathrm{K}^{-2}$ ) (see Supplemental Material [28] for details).

Carrier mobility in each sample can be quantified using three distinct experimental inputs: (i) the amplitude of magnetoresistance [Fig. 2(a)], (ii) the magnitude of the ordinary Hall conductivity and its field dependence, fitted to a two-band model [Fig. 2(b)] and yielding mobility and concentration of electrons and holes (see Ref. [28] for details), and (iii) the magnitude of zerotemperature residual resistivity. As one can see in Fig. 2(c), the mobilities extracted from the ordinary Hall conductivity are proportional to RRR, providing another check of our assumption that what distinguishes these crystals is the disorder-limited mobility of carriers. Table I lists the carrier densities and mobilities obtained by these three sets of data. We note that in the cleanest sample the zero-field mobility is 30\% larger than finite-field mobility. This is reminiscent of the field-induced reduction in mobility observed in semimetals hosting highly mobile carriers [30].

The carrier density for electrons and holes was found to be $n(\cong p)=8.7 \pm 0.3 \times 10^{19} \mathrm{~cm}^{-3}$, see Table I, and there is little variation among samples. The frequency of quantum oscillations measured on two different samples 

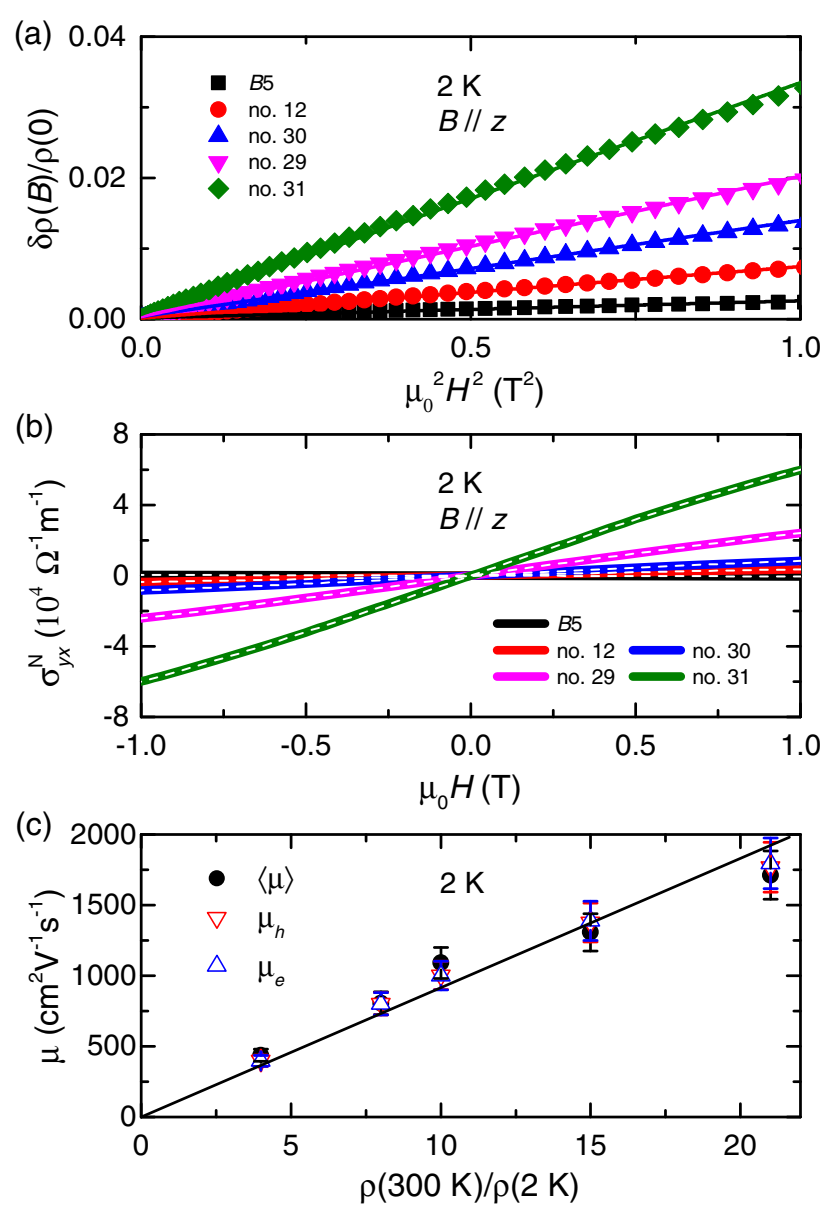

FIG. 2. Quantifying mobility. (a) Low-field magnetoresistance in five different samples as a function of $B^{2}\left(\mathrm{~T}^{2}\right)$, where $\delta \rho(B)=\rho(B)-\rho(0)$. As expected, magnetoresistance is quadratic in field and is larger in cleaner samples. (b) Low-field ordinary Hall conductivity. Dashed lines show fits to a two-band model. (c) Mobility of electrons and holes (extracted from Hall conductivity), the average mobility (extracted from magnetoresistance) as a function of RRR of each sample. They scatter around solid line with zero intercept.

(see Ref. [28]) was found to be virtually identical. The departure from compensation $2[n p /(n+p)]$ was found to be between 0.002 and and 0.04 . Thus, the large (450\%)

TABLE I. Electron (hole) Carrier density $[n(p)]$ (in units of $10^{19} \mathrm{~cm}^{-3}$ ) together with their mobilities $\left[\mu_{e}\left(\mu_{h}\right)\right]$ (in units of $\mathrm{cm}^{2} \mathrm{~V}^{-1} \mathrm{~s}^{-1}$ ) in different samples. They were extracted using a two-band fit of the ordinary Hall conductivity. The average mobility $\langle\mu\rangle$ is extracted from fitting magnetoresisitance by $[\rho(B)-\rho(0)] / \rho(0) \propto(\langle\mu\rangle B)^{2}$. The average zero-field mobility $\left\langle\mu_{0}\right\rangle$ is extracted from residual resistivity $\rho_{0}$.

\begin{tabular}{lrrcccccr}
\hline \hline Sample & RRR & Grown & $p$ & \multicolumn{1}{c}{$n$} & \multicolumn{1}{c}{$\mu_{h}$} & \multicolumn{1}{c}{$\mu_{e}$} & \multicolumn{1}{c}{$\langle\mu\rangle$} & $\left\langle\mu_{0}\right\rangle$ \\
\hline B5 & 4 & flux & 8.74 & 8.78 & 399 & 397 & 437 & 460 \\
No. 12 & 8 & CVT & 8.74 & 8.72 & 802 & 801 & 805 & 750 \\
No. 30 & 10 & CVT & 8.73 & 8.68 & 1003 & 1001 & 1091 & 989 \\
No. 29 & 15 & CVT & 8.91 & 8.71 & 1377 & 1386 & 1307 & 1542 \\
No. 31 & 21 & CVT & 8.77 & 8.42 & 1768 & 1796 & 1712 & 2260 \\
\hline \hline
\end{tabular}

change in mobility between the dirtiest and the cleanest sample is accompanied by a negligibly small shift in the chemical potential. Given that the samples remain compensated with an accuracy well below 10\% and the typical Fermi energy of electron and pockets is $40 \mathrm{meV}$ [28], it is safe to assume that any shift in chemical potential is less than $4 \mathrm{meV}$. The identity of defects is yet to be pinned down, but sulfur vacancies is one possibility.

\section{EVOLUTION OF ANOMALOUS AND ORDINARY RESPONSES WITH DISORDER}

The field dependence of Hall conductivity $\sigma_{y x}$ and the Nernst signal $S_{y x}$ in sample no. 29 at different temperatures are shown in Figs. 3(a) and 3(b). As reported previously $[12,25,26]$, there is a large hysteretic jump in both coefficients in the ferromagnetic state of $\mathrm{Co}_{3} \mathrm{Sn}_{2} \mathrm{~S}_{2}$. The magnitude of the anomalous Hall conductivity is only slightly larger than what was observed in bcc iron [10]. On the other hand, the anomalous Hall angle becomes as large as 20\%, which as previously noticed [25] is a record value. This arises because of the low longitudinal conductivity in this dilute metal.

In Figs. 3(c) and 3(d), one can see how disorder affects the two transport coefficients. In the Hall channel, the most
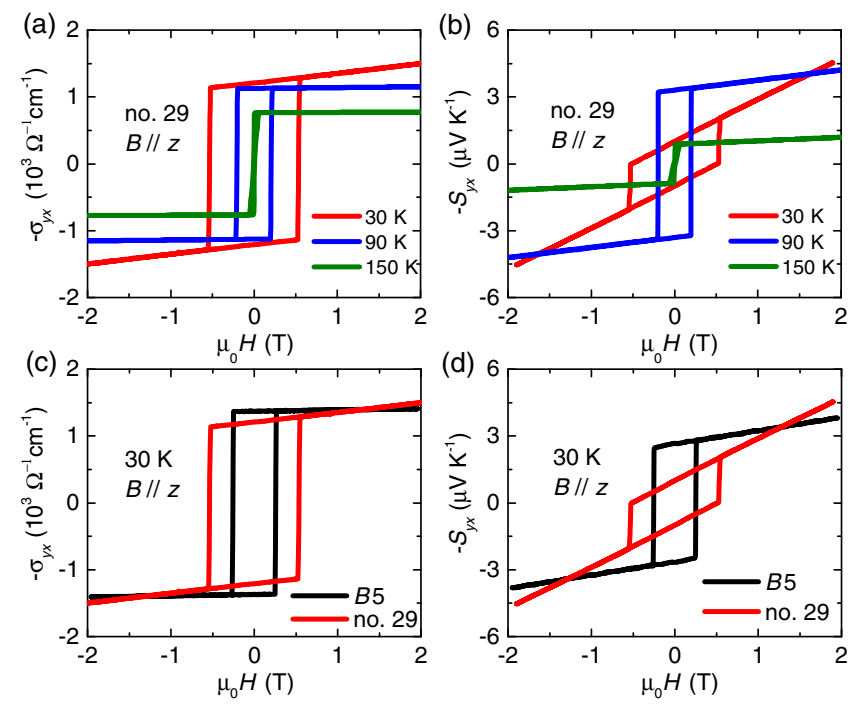

FIG. 3. Temperature-dependent AHE and ANE and their response to disorder. Top: Temperature dependence. (a) The Hall conductivity as a function of magnetic field at three different temperatures. (b) The Nernst coefficient as a function of magnetic field in at three different temperatures. Note the emergence of a hysteretic loop and anomalous responses in the ferromagnetic state. Bottom: The effect of disorder. (c) The Hall conductivity compared in two samples (the clean no. 29 and the dirty B5) at $30 \mathrm{~K}$. (d) The Nernst coefficient in the same two samples at the same temperature. The jump, which represents the anomalous component, is larger in the dirtier sample, but the slope, which represents the ordinary component, is larger in the clean sample. Note the wider hysteretic loop in the cleaner sample in both Hall and Nernst data. 

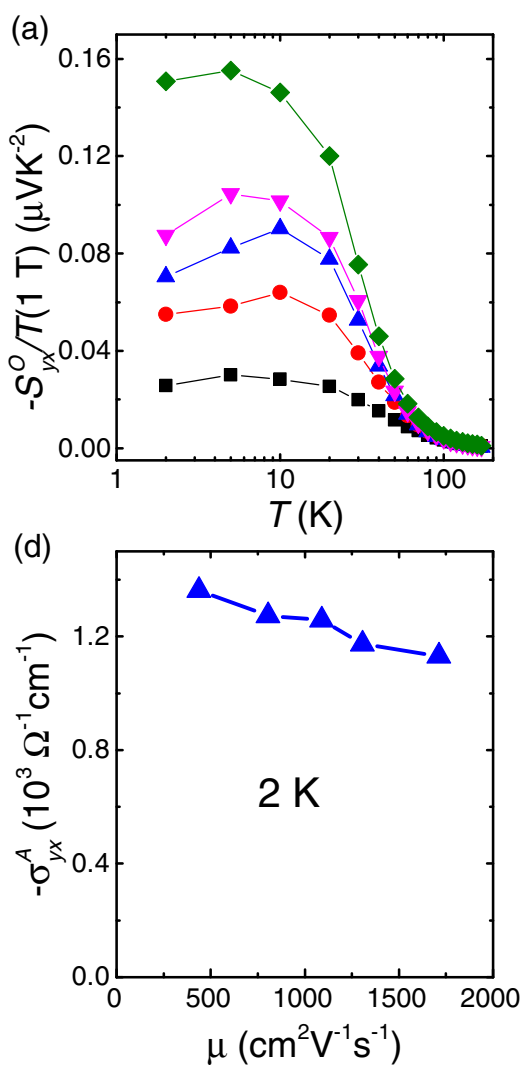
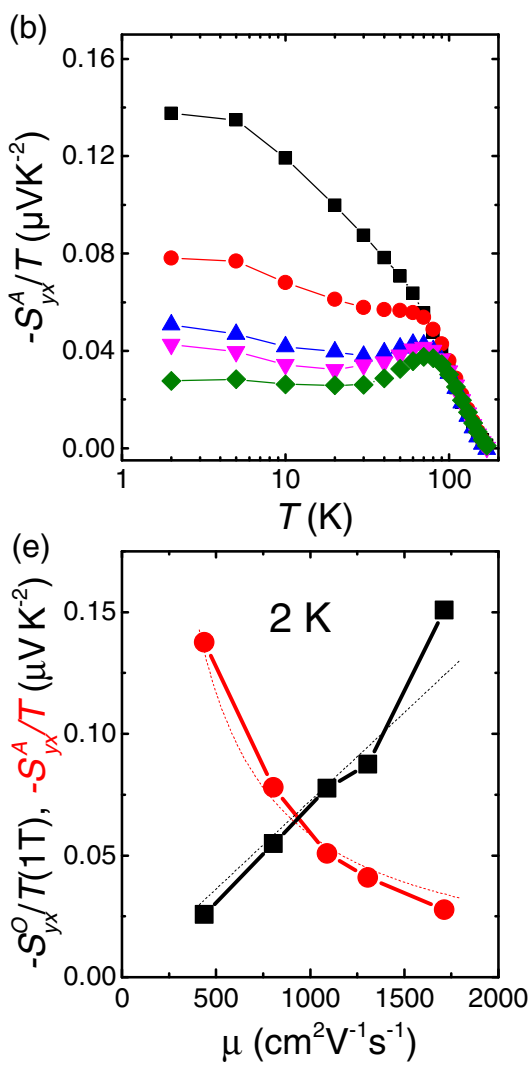
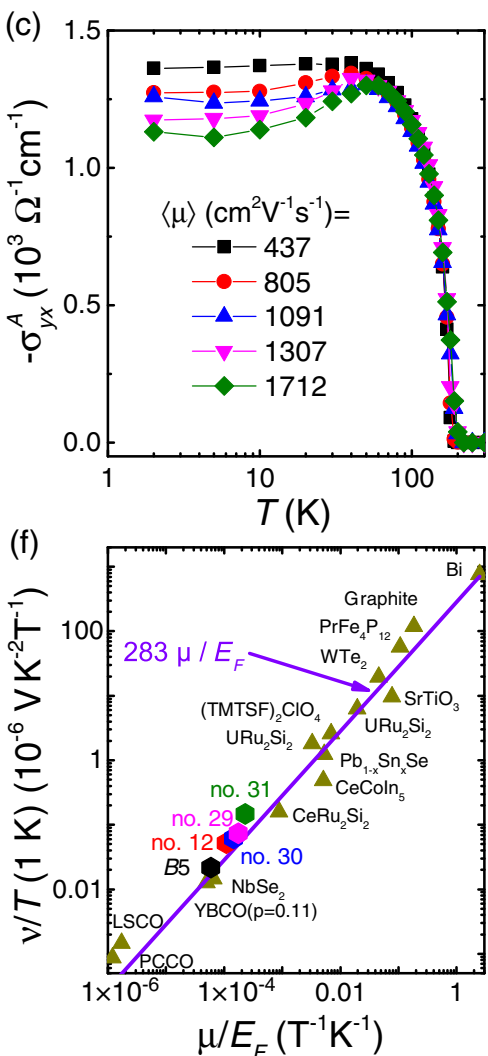

FIG. 4. Opposite evolution of ordinary and anomalous Nernst effects with disorder. Temperature dependence of the ordinary Nernst effect $S_{y x}^{O} / T$ (a), the anomalous Nernst effect $S_{y x}^{A}$ (b), and the anomalous Hall conductivity $\sigma_{y x}^{A}$ (c) in five different samples with different mobilities as defined in (a). (d) Low-temperature AHC modestly decreases with increasing mobility. (e) Variation of $S_{y x}^{O}$ and $S_{y x}^{A}$ with mobility. The first is proportional to mobility (dashed black line), but the second proportional to the inverse of mobility (dashed red line). (f) The ordinary Nernst coefficient $\nu=S_{x y}^{O} / B$ at $2 \mathrm{~K}$ compared with other solids. The solid line represents the amplitude expected in the semiclassical picture $\left(\nu / T=\left(\pi^{2} / 3\right)\left(k_{B} / e\right)\left(\mu / E_{F}\right)\right)[3]$.

visible difference between a clean and a dirty sample is the width of the hysteresis loop. There is a difference in the slope (which represents the ordinary component of the Hall conductivity set by mobility), but a very slight variation in the height of the jump (which quantifies the size of the anomalous component). In the case of the Nernst response, the difference is much more striking [Fig. 3(d)]. The cleaner sample presents a much smaller jump pointing to the amplification of the anomalous component with disorder.

We carried out an extensive set of measurements on the five samples with different mobilities in order to see how the transport coefficients evolve with temperature. A slightly attenuated disorder dependence is visible in the case of $\sigma_{y x}^{A}$ in Fig. 4(c). The fivefold increase in mobility leads to a $10 \%$ decrease in the magnitude of low-temperature $\sigma_{y x}^{A}[$ Fig. 4(d)]. Figure 4(b) shows the temperature dependence of $S_{y x}^{A}$ extracted from the jump. Figure 4(c) shows the same for ONE $S_{y x}^{O} / T$ in different samples, extracted from the $S_{y x}$ following its anomalous jump. Comparing the two plots, one can easily see the opposing tendencies. In the low-temperature limit, ONE becomes larger in the clean samples while the opposite is true for ANE. Plotting the low-temperature $S_{y x}^{A}$ and $S_{y x}^{O}$ as a function of mobility [Fig. 4(e)] reveals a striking contrast. One scales with $\mu$, while the other scales with $\mu^{-1}$. As one can see in Fig. 4(f), which compares the magnitude of ONE in the system under study with other solids, the magnitude of ONE is consistent with what is expected in a semiclassical picture given the mobility and the Fermi energy. Let us now turn our attention to the magnitude of ANE.

\section{WHAT SETS THE AMPLITUDE OF THE INTRINSIC ANOMALOUS NERNST EFFECT?}

At first sight, one may think that the amplification of the anomalous Nernst response by disorder points to an extrinsic origin. We now proceed to show that the opposite is true and this is a signature of intrinsic Berry-curvaturebased origin. We begin by recalling the expression for Hall conductivity for a two-dimensional circular isotropic Fermi surface: 


$$
\sigma_{x y}^{2 \mathrm{D}}=\frac{n e \mu}{1+\mu^{2} B^{2}} \mu B=\frac{e^{2}}{h} \frac{\ell^{2}}{\ell_{B}^{2}}\left[\frac{1}{1+\ell^{2} k_{F}^{-2} \ell_{B}^{-4}}\right] .
$$

Here, $\ell$ is the mean-free path, $k_{F}\left(\lambda_{F}\right)$ is the Fermi wave vector (wavelength), and $\ell_{B}=(\hbar / e B)^{1 / 2}$ is the magnetic length. We neglect numerical factors and focus on physical parameters. Two extreme limits, the low field $(\mu B \ll 1)$ and the high field $(\mu B \gg 1)$ are to be distinguished:

$$
\begin{gathered}
\sigma_{x y}^{\mathrm{LF}} \approx \frac{e^{2}}{h} \frac{\ell^{2}}{\ell_{B}^{2}}, \\
\sigma_{x y}^{\mathrm{HF}} \approx \frac{e^{2}}{h} \frac{\ell_{B}^{2}}{\lambda_{F}^{2}} .
\end{gathered}
$$

In the high-field limit quantum effects dominate. The latter expression provides the basis for the often-used expression for the anomalous Hall conductivity:

$$
\sigma_{x y}^{A}=-\frac{e^{2}}{\hbar} \int_{\mathrm{BZ}} \frac{d^{D} k}{(2 \pi)^{D}} f(k) \Omega_{B}(k) \approx-\frac{e^{2}}{\hbar}\left\langle\frac{\Omega_{B}}{\lambda_{F}^{2}}\right\rangle .
$$

For simplicity, we focus on the two-dimensional case $(D=2)$ and assume that $\left\langle\Omega_{B} / \lambda_{F}^{2}\right\rangle$ represents the summation of the Berry curvature $\Omega_{B}(k)$ over the relevant cross section of the Fermi surface. $\Omega_{B}$ has replaced the square of the magnetic length $\ell_{B}^{2}$. Berry curvature is indeed a fictitious magnetic field.

We turn our attention to the off-diagonal thermoelectric conductivity $\alpha_{x y}$. According to the Mott relation, it quantifies the change in $\sigma_{x y}$ caused by an infinitesimal shift in the chemical potential [31]. Thus, if the mean-free path were energy independent, there would be no low-field $\alpha_{x y}$. However, in any realistic metal, scattering is energy dependent and, therefore (see Supplemental Material [28]),

$$
\alpha_{x y}^{\mathrm{LF}} \approx \frac{e k_{B}}{h} \frac{\ell^{2}}{\ell_{B}^{2}} \frac{\lambda_{F}^{2}}{\Lambda^{2}}
$$

Here, $\Lambda=\sqrt{\left(h^{2} / 2 \pi m^{*} k_{B} T\right)}$ is the de Broglie thermal wave vector. The experimentally measured Nernst signal, $S_{y x}=\left(E_{y} / \nabla_{x} T\right)$, is simply the ratio of $\alpha_{x y}$ to longitudinal resistivity (in the small Hall angle limit). Thus,

$$
S_{x y}^{\mathrm{LF}} \approx \frac{k_{B}}{e} \frac{\ell}{\ell_{B}^{2}} \frac{\lambda_{F}^{3}}{\Lambda^{2}}
$$

Thus, the ordinary Nernst signal is proportional to the mean-free path. Note that this equation is identical to the statement that the Nernst signal at low temperatures is set by the ratio of mobility to the Fermi energy [3], which is the case in numerous metals [Fig. 4(f)]. Let us now consider the high-field regime. In this limit, it is the energy dependence of $\lambda_{F}$ (and not $\ell$ ) that matters. Using Eq. (3) and the Mott relation, one finds (see Ref. [28])

$$
\alpha_{x y}^{\mathrm{HF}} \approx \frac{e k_{B}}{h} \frac{\ell_{B}^{2}}{\Lambda^{2}}
$$

In analogy with $\sigma_{x y}^{A}$, this expression can be used to quantify the magnitude of $\alpha_{x y}^{A}$ caused by the Berry curvature fictitious field:

$$
\alpha_{x y}^{A} \approx \frac{e k_{B}}{h}\left\langle\frac{\Omega_{B}}{\Lambda^{2}}\right\rangle .
$$

We see that $\alpha_{x y}^{A}$ is also set by the summation of the Berry curvature over a cross section of the Fermi surface, with the thermal wavelength replacing the Fermi wavelength [31]. The anomalous Nernst effect would be

$$
S_{x y}^{A} \approx \frac{k_{B}}{e} \frac{1}{k_{F} \ell}\left\langle\frac{\Omega_{B}}{\Lambda^{2}}\right\rangle .
$$

This equation implies a $S_{x y}^{A}$ inversely proportional to the mean-free path. Thus, both the correlation of the ONE and the anticorrelation of the ANE with the carrier mobility can be explained from the Berry-curvature point of view.

Note that this is a single-band approach. The solid under study has multiple Fermi surface sheets, as implied by our detailed theoretical calculations and our experimental observation of two distinct frequencies in the Shubnikov-de Haas data (see Supplemental Material [28]). Nevertheless, the bulk of our experimental results can be understood within this single-band picture.

\section{ANOMALOUS OFF-DIAGONAL THERMOELECTRIC CONDUCTIVITY $\alpha_{x y}^{A}$ : THEORY AND EXPERIMENT}

Previous studies of the anomalous Nernst effect in this system $[12,14]$ reported on the magnitude of the anomalous off-diagonal thermoelectric conductivity $\alpha_{x y}^{A}(T)$. As we see below, our data and analysis lead us to a different picture from those.

The anomalous off-diagonal thermoelectric conductivity is linked to the anomalous Nernst coefficient $S_{x y}^{A}$ through three other coefficients, which are the anomalous Hall conductivity $\sigma_{x y}^{A}$, the (ordinary) longitudinal conductivity $\sigma_{x x}$, and the (ordinary) Seebeck coefficient $S_{x x}$ :

$$
\alpha_{x y}^{A}=S_{x x} \sigma_{x y}^{A}+S_{x y}^{A} \sigma_{x x} .
$$

Figure 5 presents the temperature dependence of these four coefficients in the five samples. One can see how disorder affects the four different coefficients in different ways. As expected, low-temperature conductivity $\sigma_{x x}$ is damped by decreasing mean-free path [Fig. 5(a)]. On the other hand, the low-temperature Seebeck coefficient $S_{x x}$ does not vary significantly between samples with different mobilities Fig. 5(b)]. This confirms that the chemical 

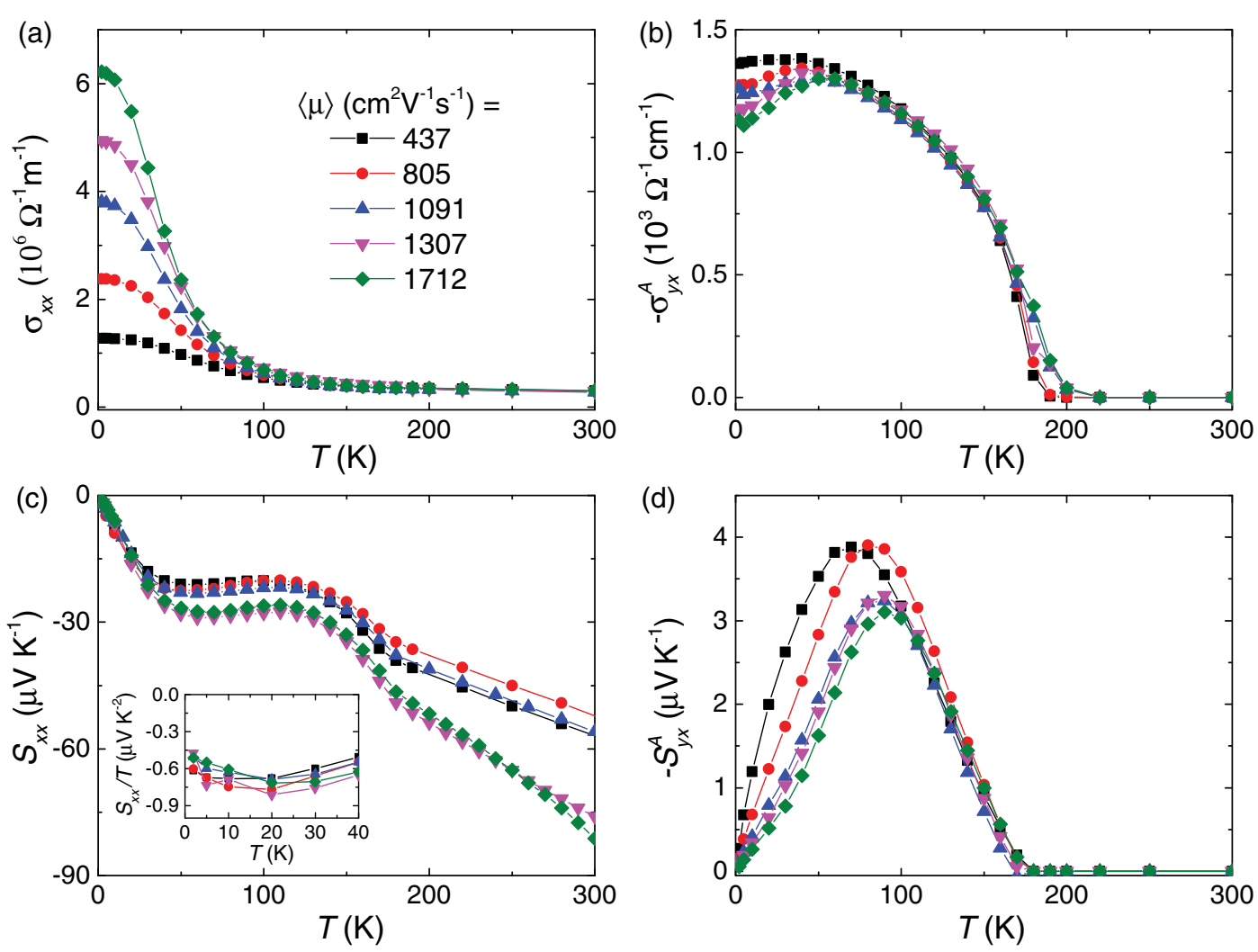

FIG. 5. Four different transport coefficients and disorder. Temperature dependence of (a) longitudinal conductivity, (b) anomalous Hall conductivity, (c) the Seebeck coefficient, and (d) the anomalous Nernst coefficient in the five samples. Note the strong variation of longitudinal conductivity and the anomalous Nernst coefficient with disorder. The anomalous Hall conductivity shows a mild variation at low temperature. As seen in the inset, the low-temperature slope of the Seebeck coefficient does not show any significant change implying that any change in the position of chemical potential is modest.

potential has not shifted significantly with the change in the mobility and the disorder is largely decoupled from doping. As seen above, disorder mildly affects $\sigma_{x y}^{A}$ [Fig. 5(c)], but drastically affects $S_{x y}^{A}$ [Fig. 5(d)].

The two terms on the right-hand side of Eq. (10) are shown in Figs. 6(a) and 6(b). As seen in the figure, the two terms are comparably large and have a maximum of the order of 3-4 $\mathrm{Am}^{-1} \mathrm{~K}^{-1}$. However, they have opposite signs, and as a result, $\alpha_{x y}^{A}$ is smaller and becomes negative below $80 \mathrm{~K}$ in all samples [see Fig. 6(c)]. Our result is different from the two previous reports $[12,14]$.

Reference [12] instead of subtracting the two components added them up. As a consequence, the peak amplitude of $\alpha_{x y}^{A}$ is much larger than what is found here or in Ref. [14]. On the other hand, the data reported in Ref. [14] match our data over a temperature range but not below $100 \mathrm{~K}$. In particular, Guin and co-workers [14] did not resolve the sign change visible in five different samples studied in this work. We can only speculate that the sign change was missed because of the scarcity of their data points below $60 \mathrm{~K}$ [see Fig. 6(c)].

As one can see in the inset of Fig. 6(c), the asymptotic zero-temperature slope of the anomalous off-diagonal thermoelectric conductivity $\alpha_{x y}^{A} / T$ in our samples is small and in the range of 0.05 to $0.10 \mathrm{Am}^{-1} \mathrm{~K}^{-2}$. The dispersion among samples reflects the accumulated uncertainties in four distinct measurements and possibly some slight yet finite change in chemical potential. What we can firmly say is that the low-temperature $\alpha_{x y}^{A}$ is negative (and not positive).

In theory, we calculated the anomalous off-diagonal thermoelectric coefficient using [4]

$$
\alpha_{x y}^{A}(T, \mu)=-\frac{1}{e} \int d \epsilon \frac{\partial f(\epsilon-\mu, T)}{\partial \epsilon} \frac{\epsilon-\mu}{T} \sigma_{x y}^{A}(\epsilon) .
$$

Here, $\sigma_{x y}^{A}(\epsilon)$ is the chemical-potential-dependent anomalous Hall conductivity (AHC) at the zero temperature [see Eq. (4)], $f(\epsilon-\mu, T)$ is the Fermi-Dirac distribution function, and $\mu$ is the chemical potential. Near the zero temperature, this equation gives the Mott relation:

$$
\left.\frac{\alpha_{x y}^{A}}{T}\right|_{T \rightarrow 0}=-\frac{\pi^{2} k_{B}^{2}}{3|e|} \frac{d \sigma_{x y}^{A}}{d \mu} .
$$

We have calculated $\tilde{\sigma}_{y x}^{A}$ based on the Berry curvature extracted from the $a b$ initio calculations and further 

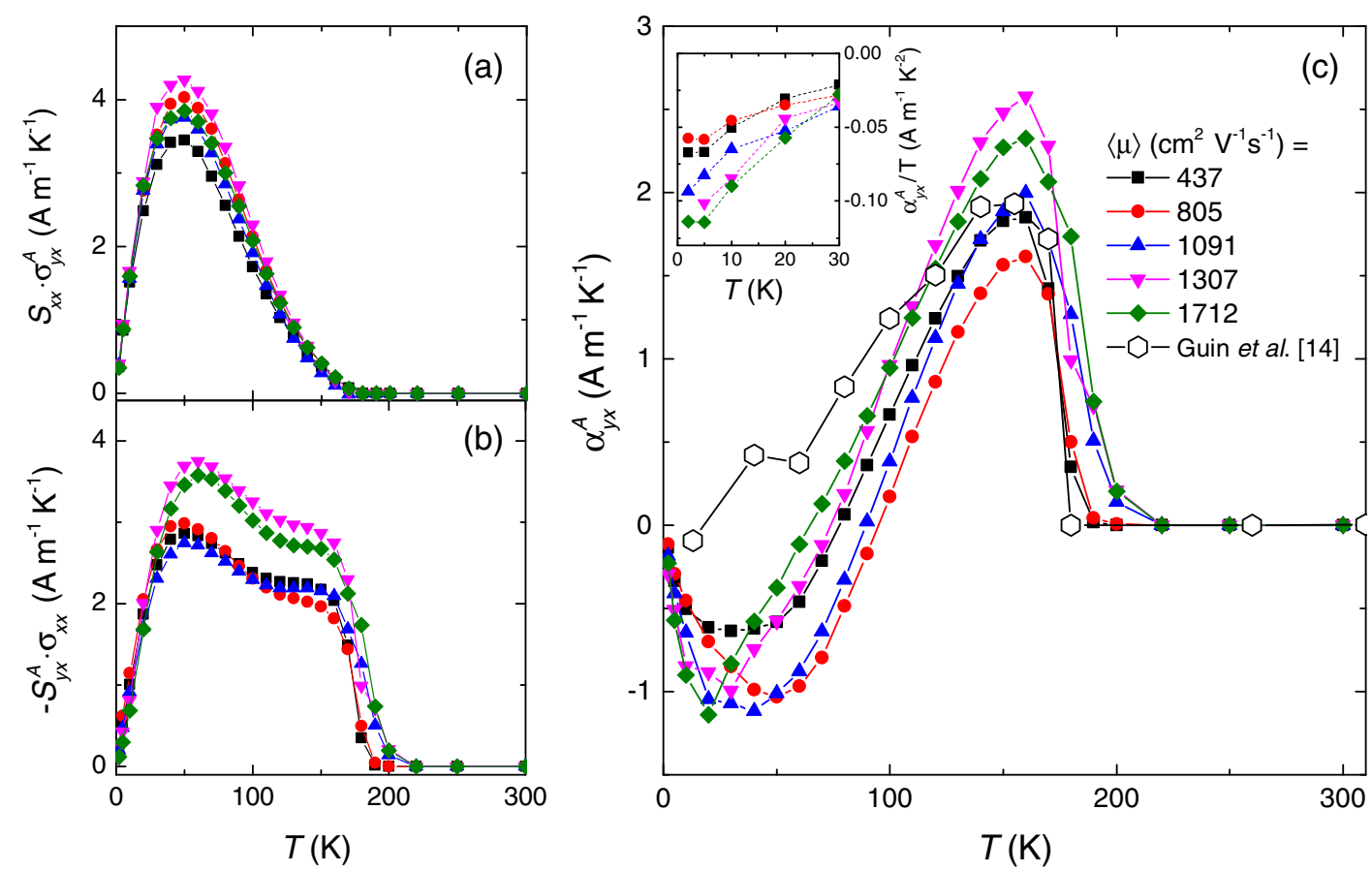

FIG. 6. Anomalous off-diagonal component of the thermoelectric tensor $\alpha_{y x}^{A}$. (a),(b) Temperature dependence of two products $S_{x x} \sigma_{y x}^{A}$ and $-S_{y x}^{A} \sigma_{x x}$. The total $\alpha_{y x}^{A}$ is the difference between these two large signals. (c). The anomalous thermoelectricity coefficient $\alpha_{y x}^{A}$ in the five samples. Also shown is the data reported by Guin et al. [14]. Note the absence of the negative slope resolved here in their data. The inset shows the $\alpha_{y x}^{A} / T$ at low temperatures to show the magnitude of the asymptotic negative slope in different samples.

obtained $\alpha_{y x}^{A}$ by Eq. (11), as shown in Fig. 7. At the charge neutral point, $\alpha_{y x}^{A}$ exhibits nearly a peak slope to $T$ near the zero temperature. This large slope is negative and is induced by the large gradient of $-\sigma_{y x}^{A}$ [see Figs. 7(a) and 7(b)]. Here $\alpha_{y x}^{A} / T$ is about $-0.03 \mathrm{Am}^{-1} \mathrm{~K}^{-2}$, smaller than but not far from the experimental value. The negative low-temperature slope exists in a wide chemical potential window $(\mu>-15 \mathrm{meV})$. This would explain why all five samples, with small variation in their chemical potential, exhibit negative slope. We can get more insight from Eq. (8). Different from $\tilde{\sigma}_{x y}^{A}$, which is determined by the Berry curvature of all bands below the Fermi surface [see Eq. (4)], $\alpha_{y x}^{A}$ is a Fermi surface property and determined by the Berry curvature $\Omega_{y x}$ at the Fermi energy [Eq. (8)]. As indicated in Fig. S7 of Supplemental Material, $\Omega_{y x}$ exhibits dominantly negative value near the charge neutral point, which is caused by anticrossing bands (E1, E2, and H2 in the Fermi surface of Fig. S7 [28]). Then large negative $\Omega_{y x}$ induces large negative $\alpha_{y x}^{A} / T$ at low temperature.

It is worth noting that $a b$ initio calculations can only reveal the low-temperature physics. Present calculations do not include the effects such as the spin fluctuation and phonons at higher temperature, which can alter the band structure and ANE. Thus, it is not surprising that the calculated $\alpha_{y x}^{A}(T)$ does not show a sharp upturn to the positive side from about $40 \mathrm{~K}$ to the magnetic transition temperature as seen in Fig. 6.
We note that Ref. [14] invoked a shift of $-80 \mathrm{meV}$ in the chemical potential in order to explain a presumably positive $\alpha_{x y}^{A}$ at higher temperature. Given the small size of the Fermi surface pockets and their Fermi energies, such a shift in the chemical potential would imply a large departure from electron-hole compensation (see Supplemental Material for the chemical-potential-dependent carrier densities [28]), which would contradict their Hall conductivity data, which are similar to ours. We conclude that the agreement between our theory and experiment is such that there is no need to invoke a huge, uncontrolled and unidentified doping to explain an $\alpha_{x y}^{A}$ of opposite sign.

Comparing the details of our experimental data with theory, we can see that the amplitude of the experimental $\tilde{\sigma}_{y x}^{A}$ [Figs. 4(d) and 6(c)] is in quantitative agreement with theory [Fig. 7(a)]. On the other hand, the experimental $\alpha_{x y}^{A}$ is $2-4$ times larger than the theoretical one. Since $\alpha_{x y}$ is the energy derivative of $\sigma_{x y}$, this indicates that theory underestimates the sensitivity of the AHE to a slight shift of the chemical potential. The observed variation of $\tilde{\sigma}_{y x}^{A}$ [200 $\Omega \mathrm{cm}^{-1}$, see Fig. 6(c)] among the five observed samples is consistent with this interpretation. If this variation is caused by a $4 \mathrm{meV}$ shift in the chemical potential, then the Mott relation would imply an $\alpha_{y x}^{A} / T$ as large as $0.12 \mathrm{Am}^{-1} \mathrm{~K}^{-2}$, close to the experimentally observed value of $\alpha_{x y}^{A} / T \approx 0.09 \pm 0.03 \mathrm{Am}^{-1} \mathrm{~K}^{-2}$ [Fig. 7(c)]. This provides further proof that the shift in 

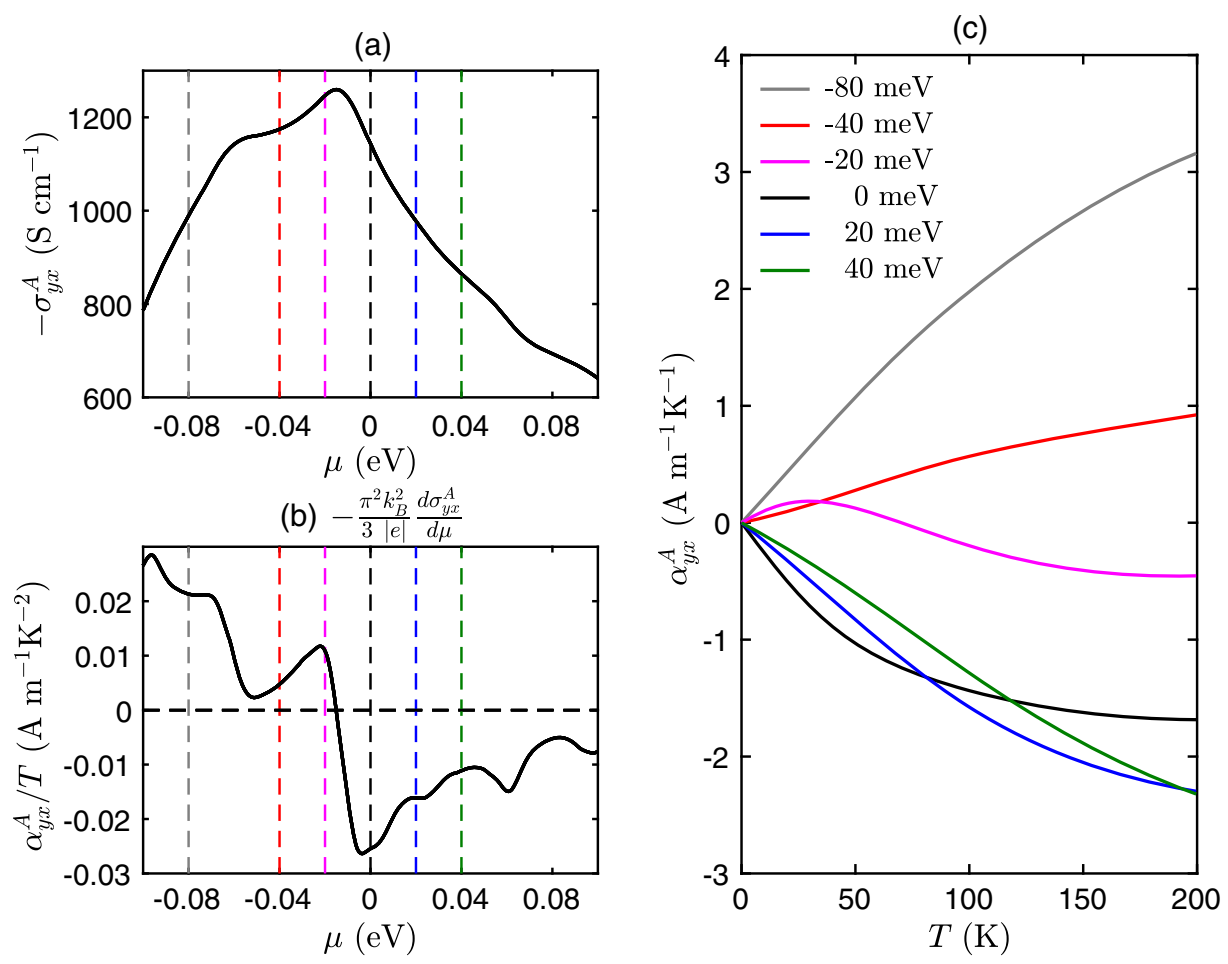

FIG. 7. Theoretical $\sigma_{y x}^{A}$ and $\alpha_{y x}^{A}$. (a) Anomalous Hall conductivity at $0 \mathrm{~K}$ as varying the chemical potential $\mu$. The charge neutral point is set to zero. (b) The slope of $\alpha_{y x}^{A}$ to $T$ in the Mott relation [Eq. (12)]. (c) Temperature dependence of $\alpha_{y x}^{A}$ at different chemical potentials.

chemical potential is modest and largely decoupled from disorder.

\section{CONCLUDING REMARKS}

In summary, we performed an extensive set of electric and thermoelectric transport measurements on $\mathrm{Co}_{3} \mathrm{Sn}_{2} \mathrm{~S}_{2}$ with different impurity contents. We found that increasing the mean-free path enhances the ordinary and reduces the anomalous components of the Nernst coefficients. Both theory and experiment find that this solid has a complex Fermi surface with multiple electron and hole pockets. However, the scaling of the anomalous Nernst coefficient with the inverse of the carrier mobility, compatible with its intrinsic Berry-curvature origin, derived in a simple oneband approach persists. Our detailed analysis of the anomalous transverse thermoelectric conductivity $\alpha_{x y}^{A}$ and its two components finds that it is negative at low temperature. Both its sign and amplitude match what theory expects without invoking uncontrolled doping.

\section{ACKNOWLEDGMENTS}

This work was supported by The National Key Research and Development Program of China (Grant No. 2016YFA0401704), the National Science Foundation of China (Grants No. 11574097 and No. 51861135104) and the Fundamental Research Funds for the Central Universities (Grant No. 2019kfyXMBZ071). Z. Z. was supported by the
1000 Youth Talents Plan. K. B. was supported by China High-end foreign expert program and by the Agence Nationale de la Recherche (ANR-18-CE92-0020-01). B. Y. acknowledges the financial support by the Willner Family Leadership Institute for the Weizmann Institute of Science, the Benoziyo Endowment Fund for the Advancement of Science, Ruth and Herman Albert Scholars Program for New Scientists, and the European Research Council (ERC) under the European Union's Horizon 2020 research and innovation programme (Grant Agreement No. 815869). H. L. was supported by the National Key R\&D Program of China (Grant No. 2016YFA0300504) and the National Natural Science Foundation of China (Grants No. 11574394, No. 11774423, No. 11822412).

[1] N. Nagaosa, J. Sinova, S. Onoda, A. H. MacDonald, and N. P. Ong, Anomalous Hall Effect, Rev. Mod. Phys. 82, 1539 (2010).

[2] D. Xiao, M.-C. Chang, and Q. Niu, Berry Phase Effects on Electronic Properties, Rev. Mod. Phys. 82, 1959 (2010).

[3] K. Behnia and H. Aubin, Nernst Effect in Metals and Superconductors: A Review of Concepts and Experiments, Rep. Prog. Phys. 79, 046502 (2016).

[4] D. Xiao, Y. Yao, Z. Fang, and Q. Niu, Berry-Phase Effect in Anomalous Thermoelectric Transport, Phys. Rev. Lett. 97, 026603 (2006).

[5] W.-L. Lee, S. Watauchi, V. L. Miller, R. J. Cava, and N. P. Ong, Anomalous Hall Heat Current and Nernst Effect in the 
$\mathrm{CuCr}_{2} \mathrm{Se}_{4-x} \mathrm{Br}_{x}$ Ferromagnet, Phys. Rev. Lett. 93, 226601 (2004).

[6] T. Miyasato, N. Abe, T. Fujii, A. Asamitsu, S. Onoda, Y. Onose, N. Nagaosa, and Y. Tokura, Crossover Behavior of the Anomalous Hall Effect and Anomalous Nernst Effect in Itinerant Ferromagnets, Phys. Rev. Lett. 99, 086602 (2007).

[7] A. Sakai, Y. P. Mizuta, A. A. Nugroho, R. Sihombing, T. Koretsune, M.-T. Suzuki, N. Takemori, R. Ishii, D. NishioHamane, R. Arita, P. Goswami, and S. Nakatsuji, Giant Anomalous Nernst Effect and Quantum-Critical Scaling in a Ferromagnetic Semimetal, Nat. Phys. 14, 1119 (2018).

[8] M. Ikhlas, T. Tomita, T. Koretsune, M.-T. Suzuk, D. NishioHamane, R. Arita, Y. Otani, and S. Nakatsuji, Large Anomalous Nernst Effect at Room Temperature in a Chiral Antiferromagnet, Nat. Phys. 13, 1085 (2017).

[9] Y. Pu, D. Chiba, F. Matsukura, H. Ohno, and J. Shi, Mott Relation for Anomalous Hall and Nernst Effects in $\mathrm{Ga}_{1-x} \mathrm{Mn}_{x}$ As Ferromagnetic Semiconductors, Phys. Rev. Lett. 101, 117208 (2008).

[10] X. Li, L. Xu, L. Ding, J. Wang, M. Shen, X. Lu, Z. Zhu, and K. Behnia, Anomalous Nernst and Righi-Leduc Effects in $\mathrm{Mn}_{3} \mathrm{Sn}$ : Berry Curvature and Entropy Flow, Phys. Rev. Lett. 119, 056601 (2017).

[11] L. Xu, X. Li, X. Lu, C. Collignon, H. Fu, B. Fauqué, B. Yan, Z. Zhu, and K. Behnia, Finite-Temperature Violation of the Anomalous Transverse Wiedemann-Franz Law in Absence of Inelastic Scattering, arXiv:1812.04339.

[12] H. Yang, W. You, J. Wang, J. Huang, C. Xi, C. Cao, M. Tian, Z.-A. Xu, J. Dai, and Y. Li, Giant Anomalous Nernst Effect in the Magnetic Weyl Semimetal $\mathrm{Co}_{3} \mathrm{Sn}_{2} \mathrm{~S}_{2}$, arXiv:1811.03485.

[13] C. Wuttke, F. Caglieris, S. Sykora, F. Scaravaggi, A. U. B. Wolter, K. Manna, V. Süss, C. Shekhar, C. Felser, B. Büchner, and C. Hess, Berry Curvature Unravelled by the Nernst Effect in $\mathrm{Mn}_{3} \mathrm{Ge}$, Phys. Rev. B 100, 085111 (2019).

[14] S. N. Guin, P. Vir, Y. Zhang, N. Kumar, S. J. Watzman, C. Fu, E. Liu, K. Manna, W. Schnelle, J. Gooth, C. Shekhar, Y. Sun, and C. Felser, Zero-Field Nernst Effect in a Ferromagnetic Kagome-Lattice Weyl-Semimetal $\mathrm{Co}_{3} \mathrm{Sn}_{2} \mathrm{~S}_{2}$, Adv. Mater. 31, 1806622 (2019).

[15] X. Li, L. Xu, H. Zuo, A. Subedi, Z. Zhu, and K. Behnia, Momentum-Space and Real-Space Berry Curvatures in $\mathrm{Mn}_{3} \mathrm{Sn}$, SciPost Phys. 5, 063 (2018).

[16] K. Sugii, Y. Imai, M. Shimozawa, M. Ikhlas, N. Kiyohara, T. Tomita, M.-T. Suzuki, T. Koretsune, R. Arita, S. Nakatsuji, and M. Yamashita, Anomalous Thermal Hall Effect in the Topological Antiferromagnetic State, arXiv:1902.06601.

[17] N. P. Armitage, E. J. Mele, and A. Vishwanath, Weyl and Dirac Semimetals in Three-Dimensional Solids, Rev. Mod. Phys. 90, 015001 (2018).

[18] B. Yan and C. Felser, Topological Materials: Weyl Semimetals, Annu. Rev. Condens. Matter Phys. 8, 337 (2017).
[19] K. Behnia, The Nernst Effect and the Boundaries of the Fermi Liquid Picture, J. Phys. Condens. Matter 21, 113101 (2009).

[20] P. Vaqueiro and G. G. Sobany, A Powder Neutron Diffraction Study of the Metallic Ferromagnet $\mathrm{Co}_{3} \mathrm{Sn}_{2} \mathrm{~S}_{2}$, Solid State Sci. 11, 513 (2009).

[21] M. Holder, Y. S. Dedkov, A. Kade, H. Rosner, W. Schnelle, A. Leithe-Jasper, R. Weihrich, and S. L. Molodtsov, Photoemission Study of Electronic Structure of the Half-Metallic Ferromagnet $\mathrm{Co}_{3} \mathrm{Sn}_{2} \mathrm{~S}_{2}$, Phys. Rev. B 79, 205116 (2009).

[22] W. Schnelle, A. Leithe-Jasper, H. Rosner, F. M. Schappacher, R. Pöttgen, F. Pielnhofer, and R. Weihrich, Ferromagnetic Ordering and Half-Metallic State of $\mathrm{Sn}_{2} \mathrm{Co}_{3} \mathrm{~S}_{2}$ with the Shandite-Type Structure, Phys. Rev. B 88, 144404 (2013).

[23] J. M. D. Coey and S. Sanvito, Magnetic Semiconductors and Half-Metals, J. Phys. D 37, 988 (2004).

[24] J.-X. Yin, S. S. Zhang, G. Chang, Q. Wang, S. S. Tsirkin, Z. Guguchia, B. Lian, H. Zhou, K. Jiang, I. Belopolski, N. Shumiya, D. Multer, M. Litskevich, T. A. Cochran, H. Lin, Z. Wang, T. Neupert, S. Jia, H. Lei, and M. Z. Hasan, Negative Flat Band Magnetism in a Spin-Orbit-Coupled Correlated Kagome Magnet, Nat. Phys. 15, 443 (2019).

[25] E. Liu, Y. Sun, N. Kumar, L. Muechler, A. Sun, L. Jiao, S.-Y. Yang, D. Liu, A. Liang, Q. Xu, J. Kroder, V. Süß, H. Borrmann, C. Shekhar, Z. Wang, C. Xi, W. Wang, W. Schnelle, S. Wirth, Y. Chen, S. T. B. Goennenwein, and C. Felser, Giant Anomalous Hall Effect in a Ferromagnetic Kagome-Lattice Semimetal, Nat. Phys. 14, 1125 (2018).

[26] Q. Wang, Y. Xu, R. Lou, Z. Liu, M. Li, Y. Huang, D. Shen, $\mathrm{H}$. Weng, S. Wang, and H. Lei, Large Intrinsic Anomalous Hall Effect in Half-Metallic Ferromagnet $\mathrm{Co}_{3} \mathrm{Sn}_{2} \mathrm{~S}_{2}$ with Magnetic Weyl Fermions, Nat. Commun. 9, 3681 (2018).

[27] N. Morali, R. Batabyal, P. K. Nag, E. Liu, Q. Xu, Y. Sun, B. Yan, C. Felser, N. Avraham, and H. Beidenkopf, Fermi-Arc Diversity on Surface Terminations of the Magnetic Weyl Semimetal $\mathrm{Co}_{3} \mathrm{Sn}_{2} \mathrm{~S}_{2}$, Science 365, 1286 (2019).

[28] See Supplemental Material at http://link.aps.org/ supplemental/10.1103/PhysRevX.9.041061 for sample growth and preparation, Hall and Nernst measurements, carrier density and mobility extraction, Curie temperature and coercive field in the samples, T-square resistivity, derivation of $\alpha_{x y}$ using the Mott formula, multiplicity of the Fermi surface pockets in $\mathrm{Co}_{3} \mathrm{Sn}_{2} \mathrm{~S}_{2}$ and DFT calculation.

[29] X. Lin, B. Fauqué, and K. Behnia, Scalable $\mathrm{T}^{2}$ Resistivity in a Small Single-Component Fermi Surface, Science 349, 945 (2015).

[30] B. Fauqué, X. Yang, W. Tabis, M. Shen, Z. Zhu, C. Proust, Y. Fuseya, and K. Behnia, Magnetoresistance of Semimetals: The Case of Antimony, Phys. Rev. Mater. 2, 114201 (2018).

[31] K. Behnia, Fundamentals of Thermoelectricity (Oxford University Press, Oxford, 2015). 